\title{
Knowledge, Attitude and Practice Among Type II Diabetic Patients in Ambo University Referral Hospital, Oromia, Ethiopia
}

\author{
Agama Daba Tamiru Yazew \\ Department of Food Science and Nutrition, College of Agriculture, Shambu Campus, Wollega University, \\ Shambu, Ethiopia, Box 38, Shambu, Ethiopia
}

\begin{abstract}
Background: Diabetes is a chronic illness significantly affecting health of population throughout the world. In Africa, about 19.8 million adults are affected by diabetes and of these, more than $50 \%$ are from Nigeria, South Africa, Ethiopia and Tanzania. Knowledge is the greatest weapon in the fight against diabetes mellitus (DM). Selfmanagement of patients with type two DM largely depends on the knowledge, attitude and practices of such people regarding the condition. Objective: The main objective of this study was to assess knowledge, attitude and practices of type II diabetes (T2DM) patients attending Ambo University referral hospital, Oromia, Ethiopia. Method: An institutional based cross sectional study was conducted among randomly selected 248 T2DM patients aged $\geq 30$ years old from Ambo University Referral Hospital. Data regarding KAP of diabetic patients were collected using interview techniques. In addition, anthropometric data (body mass index and waist hip ratio) of the participants were collected and assessed. The tool for data collection contains socio demographic characteristics and medical factors. Descriptive statistics was used to set the KAP level among the respondents while logistic regression model was applied to test the association of KAP with various independent variables. Results: The mean of age among the respondents was 44( \pm 7.2$)$ years. Of 248 respondents, $105(42.3 \%)$ were from rural. Among the total 248 respondents, about 111(44.8\%), 117(47.2\%) and 121(48.8\%) were differentiated as not knowledgeable, having poor attitude and poor practice towards diabetes mellitus, respectively. Age, place of residence, level of education, average monthly income, history of diabetes and duration of living with DM were identified as factors affecting KAP towards diabetes. Both sex and occupational status were also factors affecting the knowledge level of T2DM patients, but not attitude and practice. Furthermore, knowledge level was affecting booth attitude and practice level of participants in the study area. Conclusion and Recommendation: This study found that there was a gap in knowledge, attitude and practice towards diabetes among T2DM patients in the study area. Therefore, a structured nutrition and health education should be given for T2DM patients through community based behavioral change communication to assist and improve the level of KAP towards DM in the study area.
\end{abstract}

Keywords: Type 2 diabetes mellitus, Knowledge, Attitude, Practice, Adults

DOI: $10.7176 / \mathrm{JMPB} / 66-01$

Publication date:June 30th 2020

\section{Introduction}

Diabetes is a chronic illness that significantly affecting health of the population throughout the world. It is a longterm metabolic disorder that occurs either due to inadequate insulin production by the pancreas (type 1 diabetes mellitus) or when the produced insulin is not effectively used by the body (type 2 diabetes mellitus) (WHO, 2016). Type 2 diabetes mellitus (T2DM) is the most common form of diabetes mellitus and can be controlled through healthy diet, physical activity, losing excess weight, and oral medication (CDC, 2012). Gestational diabetes mellitus occurs only during pregnancy and is a risk factor for T2DM after pregnancy (Mabaso et al., 2014). American Diabetes Association stated that DM may result from causes such as genetic defects in beta cell function, insulin action, pancreatic diseases, and drug or chemical induced diabetes mellitus such as with HIV medication (ADA, 2010).

According to the world health organization estimate, globally, the number of people with diabetes will be more than double over the next 25 years and the developing world would endure an increasingly larger burden of disease in that period (Primanda et al., 2011). This incidence might be varied between populations because of differences in genetic susceptibility and other modifiable risk factors.

Sub-Saharan Africa, like the rest of the world, is experiencing an increasing prevalence of diabetes alongside other non-communicable diseases. In Africa, according to the International Diabetes Federation (IDF) report, 19.8 million adults were estimated to have diabetes. Of this, more than $50 \%$ live in four highly populated countries namely; Nigeria, South Africa, Ethiopia and Tanzania (Cho et al., 2013). Ethiopia is at a risk of increased diabetes incidence. The number of deaths attributed to diabetes reached over 21,000 in 2007. This estimate has increased to about 25,000 in 2011 (Feleke et al., 2013). IDF also reported that about 1.9 million adults aged $20-79$ years in Ethiopia were estimated to have diabetes in 2013 (Guariguata et al., 2013). With national diabetes prevalence of $4.36 \%$ and there was about 34,262 estimated diabetes related deaths in same year (Cho et al., 2013).

Despite the availability of different treatment modalities, diabetes has remained a major cause of death and 
its burden is increasing in the developing countries including Ethiopia. IDF reported that Ethiopia ranked $3^{\text {rd }}$ among African countries with 1.4 million DM by year 2012. Worku et al., (2010) reported that the number of diabetics in Ethiopia was about 800,000 by the year 2000, and it could reach 1.8 million by 2030 . Further report in 2014 by IDF shows that about 4.9 million people adults (20-79 years) in Ethiopia live with diabetes and more than 2.9 million live with impaired glucose tolerance. Another study done in the selected hospitals of Addis Ababa reported that the prevalence of T2DM was in the range of 4.6 to 5.1\% (Belayneh et al., 2015). Moreover, a study done in Bishoftu town, Ethiopia showed that the prevalence of undiagnosed diabetes mellitus was 5\% (Megersa et al., 2013).Furthermore, a cross-sectional study done in Nekemte Referral hospital, Ethiopia also showed that there were many patients who lived with T2DM (55\%) compared to those patients with type I diabetes $(45 \%)($ Tadele et al., 2014).

Knowledge is the greatest weapon in the fight against diabetes mellitus. It is therefore imperative that people with DM and their family members understand the basic facts, such as the type they have, signs and symptoms, treatments, preventive measures, and the importance of maintaining good glycemic control (Raymond et al., 2016). Beke et al., (2013) emphasized that self-management of patients with T2DM largely depends on the KAP. A study done in Northwest Ethiopia revealed that about more than $51 \%$ and $60.1 \%$ of T2DM patients had poor knowledge and poor attitude, respectively (Achenef et al., 2015). However, studies assessing KAP towards T2DM and associated factors among type two diabetic patients are scanty in the study area. Therefore, this study was designed to assess the level of KAP of T2DM among diabetic patients and factors affecting KAP towards T2DM in the study area.

\section{Methodology}

\subsection{Description of the Study Area}

Ambo University is one of the higher public academic institutions in Ethiopia. It is located in west Showa, Ambo town, west of Addis Ababa $112 \mathrm{~km}$ and the town has a latitude and longitude of $8^{\circ} 59^{\prime} \mathrm{N}$ and $37^{\circ} 51^{\prime} \mathrm{E}$, respectively and an elevation of 2101 meters above sea level. The average annual temperature is $17.5^{\circ} \mathrm{C}$ in Ambo. The main agricultural crops grown in the areas are tef, wheat, barley, and maize, sorghum and fruit and vegetables, especially fruits obtained from market with expensive cost. According to the town municipality, more than 121,061 populations live in the town in 2019, in which males accounts for 59,322(49\%) and females 61,739(51\%).

\subsection{Study Design and Period}

An institutional based cross-sectional study was conducted among T2DM patients attending in Ambo University Referral Hospital, Oromia, Ethiopia from December 2018 to May 2019.

\subsection{Source and Study Population}

All T2DM patients' $\geq 30$ years of age and visited Ambo University Referral Hospital during the survey were the source population and all type 2 diabetic patients' $\geq 30$ years of age and randomly selected from Ambo University Referral Hospital were the study population.

\subsection{Inclusion and Exclusion Criteria}

Individuals who were diagnosed to have T2DM and aged $\geq 30$ years old were included in the study while those individuals who were chronically sick at the time of survey and aged $<30$ years old were excluded.

\subsection{Sample Size Determination}

The sample size of the study is calculated using formula for a single population proportion by considering the following assumptions:

$$
n=Z_{\alpha / 2}{ }^{2} \frac{P[1-P]}{d^{2}}
$$

Where: $n=$ required sample sizes, $Z \alpha / 2=95 \%$ confidence level $(Z=1.96), P=$ expected prevalence of poor KAP about DM was $11.2 \%, 18.1 \%$ and $33.6 \%$, respectively among T2DM patients attending Adama hospital Medical college (Abdulkadi et al., 2014) and $d=5 \%$. Therefore, the largest sample size $(n=248)$ was used with the consideration of $10 \%$ non response rate.

\subsection{Sampling Procedures}

The study samples were selected by using simple random sampling method; from subjects with type two DM $\geq 30$ years old attending the diabetic clinic follow up in Ambo University Referral Hospital. Generally, the detail of sampling technique is given as the below Fig 1. 


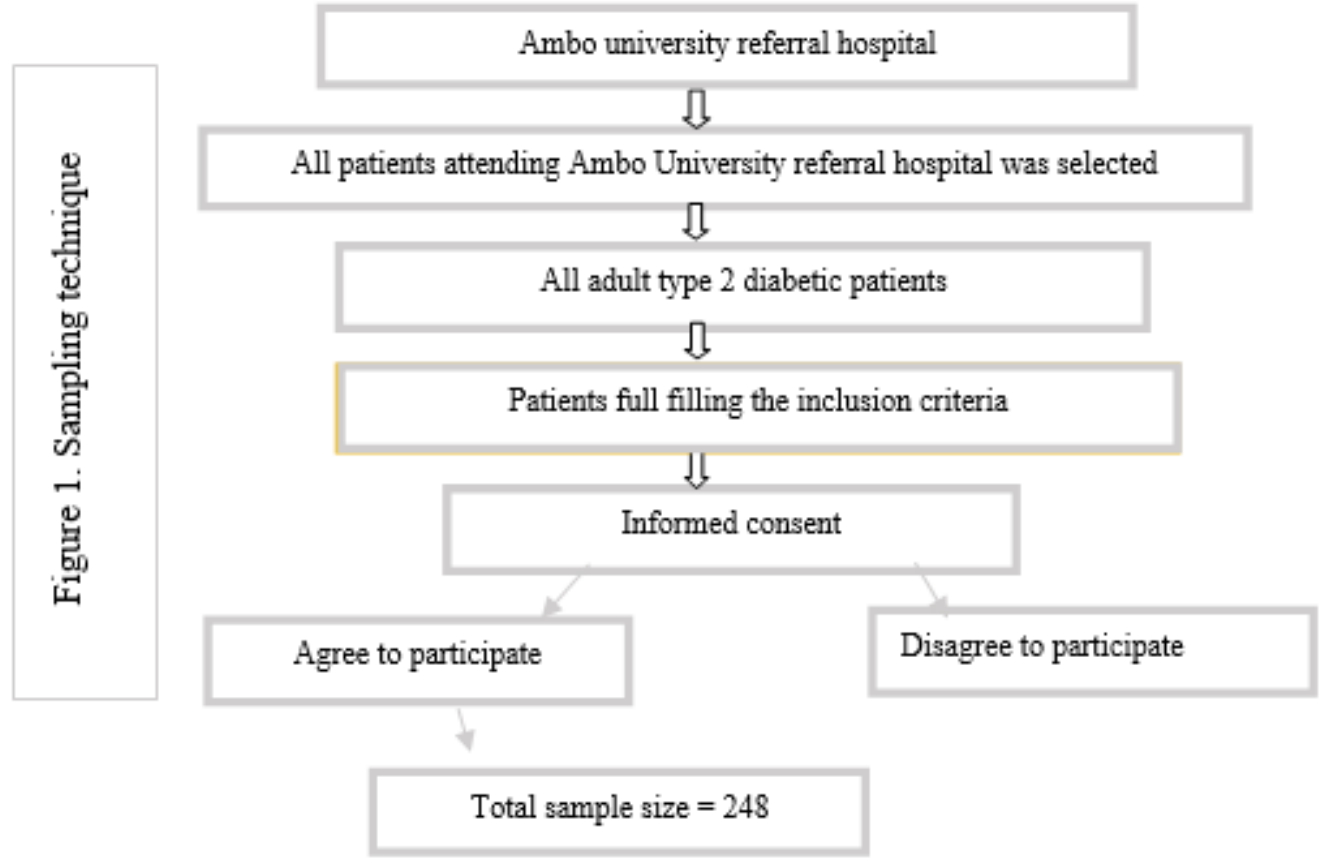

\subsection{Study Variables}

In this study, the knowledge, attitude and practices (KAP) were dependent variables while socio-demographic and economic characteristics as well as medical factors were independent variables.

\subsection{Data Collection Methods and Quality Assurance}

Data regarding KAP towards DM were collected using interview techniques and a structured questionnaire. Data were collected by four nursing diploma holder with the supervision of the researchers. To assure data quality, a training and orientation was given for the data collectors. The questionnaire was first developed in English and then translated into Afaan Oromoo and then back translated to English. The questionnaire was pre-tested in 5\% of the respondent in a health center not included in the study to check for its understandability and time required completing the questionnaire before the actual data collection begun. The completeness of the collected data was checked by the researcher daily. Anthropometric data like height, weight, waist and hip of subjects were measured. The participants BMI and Waist to Hip ration was classified using WHO standards as follows; underweight BMI $<18.5$, normal BMI 18.5 to 24.9 , overweight $\mathrm{BMI} \geq 25.0$ and $<29.9$ and obese $\mathrm{BMI} \geq 30.0$ (WHO, 2008). A waist hip ratio greater than 0.90 for men or greater than 0.85 for women also indicates risk factors for type two diabetic mellitus (WHO, 2008).

\subsection{Statistical analysis}

Data were analyzed using SPSS statistical software version 21.0. Descriptive statistics was used and presented using tables, graphs and percentages. Logistic regression model was used to identify factors associated to KAP level of the respondents. P-value less than 0.05 were considered as statistically significant. The degree of association between dependent and independent variables was reported using Adjusted Odds Ratio (AOR) and 95\% CI.

\subsection{Ethical consideration}

Ethical approval was obtained from Institutional Review Board (IRB) of the Addis Ababa University, College of Natural and Computational Sciences. A formal letter of permission was obtained from Ambo University Referral Hospital. After explaining the purpose of the study, verbal consent was obtained from each study participant. Participants were informed that participation is on voluntary basis and that they can withdraw at any time if they are not comfortable. Personal identifiers were not included in the questionnaires to ensure participants' confidentiality. At the end, the nutrition and health education on prevention, control and management of T2DM was given to the respondents to improve their level of KAP towards DM. 


\section{Results and Discussion}

\subsection{Results}

\subsubsection{Socio-demographic characteristics}

The male to female ratio in this study was 1.88 .The mean of age respondents was $44( \pm 7.2)$. Of 248 respondents, $105(42.3 \%)$ were from rural and $143(57.7 \%)$ were from urban areas. Among the study participants, 57(23\%) were not having formal education and $73(29.44 \%)$ of respondents were from having $\leq 500$ Ethiopian birr monthly family income (Table 1).

Table 1. Socio demographic characteristics of type 2 diabetic patients attending Ambo, Ethiopia, 2019

\begin{tabular}{|c|c|c|c|}
\hline Variable & Level & No. & $\%$ \\
\hline \multirow[t]{3}{*}{ Age (in year) } & $\leq 35$ & 28 & 11.3 \\
\hline & $36-55$ & 197 & 79.4 \\
\hline & $>55$ & 23 & 9.3 \\
\hline \multirow[t]{2}{*}{ Sex } & Male & 162 & 65.3 \\
\hline & Female & 86 & 34.7 \\
\hline \multirow[t]{2}{*}{ Place of residence } & Rural & 105 & 42.3 \\
\hline & Urban & 143 & 57.7 \\
\hline \multirow[t]{4}{*}{ Religion } & Orthodox & 162 & 65.3 \\
\hline & Protestant & 56 & 22.6 \\
\hline & Waaqeffataa & 15 & 6.0 \\
\hline & Muslim & 15 & 6.0 \\
\hline \multirow[t]{4}{*}{ Respondent education level } & No formal education & 57 & 23.0 \\
\hline & Primary education & 87 & 35.1 \\
\hline & Secondary and preparatory & 36 & 14.5 \\
\hline & College and above & 68 & 27.4 \\
\hline \multirow[t]{4}{*}{ Marital status } & Married & 221 & 89.1 \\
\hline & Divorced & 3 & 1.2 \\
\hline & Single & 13 & 5.2 \\
\hline & Widowed & 11 & 4.4 \\
\hline \multirow[t]{3}{*}{ Living status } & Living alone & 13 & 5.2 \\
\hline & Living with wife/husband & 222 & 89.5 \\
\hline & Living with others & 13 & 5.2 \\
\hline \multirow[t]{4}{*}{ Occupational status } & Housewife only & 32 & 12.9 \\
\hline & Farmer & 92 & 37.1 \\
\hline & Merchants & 40 & 16.1 \\
\hline & Gov't and private employed & 84 & 33.9 \\
\hline \multirow[t]{4}{*}{ Family income (Birr) } & $\leq 500$ & 73 & 29.4 \\
\hline & $501-1000$ & 61 & 24.6 \\
\hline & $1001-2000$ & 18 & 7.3 \\
\hline & $>2000$ & 96 & 38.7 \\
\hline
\end{tabular}

\subsubsection{Anthropometric characteristics of the respondents}

About 9(3.6\%) and 32(12.9\%) respondents were with underweight and overweight, respectively. Additionally, the mean of waist to hip ration (WHR) was 0.94 vs. 0.87 for male and female, respectively. Moreover, about 202(81.5\%) respondents had waist-hip ratio above cut-off points (Table. 2).

Table 2. Body mass index and waist hip ratio status of respondents (T2DM), Ambo university referral hospital, Oromia, Ethiopia, 2019

\begin{tabular}{lll}
\hline Variable & Level (category) & No.(\%) \\
\hline BMI & Underweight & $9(3.6)$ \\
& Normal & $207(83.5)$ \\
& Overweight & $32(12.9)$ \\
\hline WHR & Normal & $46(18.5)$ \\
& At risk & $202(81.5)$ \\
\hline
\end{tabular}

$\mathrm{BMI}=$ Body mass index, $\mathrm{WHR}=$ waist hip ratio

\subsubsection{History and duration of diabetes mellitus of participants}

Table 3 below shows that, among the respondents 77(31\%) were not having DM history or they do not know their family diabetes history and about $171(69 \%)$ were from those having family diabetic history, while the rests were from either father or mother was diabetic. More than two third of the study participants were with DM for 13 and above months (table 4). 
Table 3 Frequency distribution of DMH and DDM of respondent's (T2DM), Ambo university referral hospital, Oromia, Ethiopia, 2019

\begin{tabular}{llc}
\hline Variables & Level & No. (\%) \\
\hline DMH & No diabetic history & $77(31)$ \\
& Having DM history & $171(69)$ \\
\hline DDM & $1-6$ months & $36(14.5)$ \\
& $7-12$ months & $40(16.1)$ \\
& 13 months and above & $172(69.4)$ \\
\hline
\end{tabular}

$\mathrm{DMH}=$ Diabetic history, DDM= Duration of DM

3.1.4 Knowledge of participants towards diabetes mellitus

Table 4 below shows that the responses of participants on different questions addressing the knowledge towards DM. Participants responded as diabetes is a condition of insufficient insulin production in the body (47.6\%), as $\mathrm{DM}$ is not curable (85.9\%) and as DM is a condition of high level of sugar in the blood (85.1\%). Regarding signs and symptoms of diabetes participants responded as, frequent hunger $(87.1 \%)$, frequent thirst $(83.9 \%)$, frequent urination (43.6\%), high blood sugar (87.9\%), blurred vision $(70.9 \%)$. Regarding knowledge of associated factors, in this study, respondents stated that as age (33\%), genetic $(77.4 \%)$, lack of physical exercise $(92.7 \%)$, lack of nutrient intake $(39.9 \%)$ and obesity $(44.7 \%)$ could be risk factors to develop diabetes. On knowledge of complications, the response was blindness $(65.3 \%)$, heart problem $(84.7)$, and brain diseases $(81.1 \%)$ were major complication of diabetes identified by cases and controls, respectively. Additionally, participants also described that diabetes can be managed by insulin injection (100\%), regular exercise $(100 \%)$ and practices healthy diet $(91.1 \%)$ (Table 4).

Table 4 Frequency distribution of participant's response of knowledge towards diabetes mellitus, Ambo university referral hospital, Oromia, Ethiopia, 2019

\begin{tabular}{|c|c|c|}
\hline \multirow[t]{2}{*}{ Questions } & \multicolumn{2}{|c|}{ Response (YES) } \\
\hline & n & $\%$ \\
\hline \multicolumn{3}{|l|}{ What is DM } \\
\hline $\mathrm{DM}$ is a condition of insufficient insulin production & 118 & 47.6 \\
\hline $\mathrm{DM}$ is a condition of high level of sugar in the blood & 211 & 85.1 \\
\hline $\mathrm{DM}$ is not curable disease & 213 & 85.9 \\
\hline $\mathrm{DM}$ is a condition of the body which not responding for insulin & 88 & 35.5 \\
\hline DM is diseases which affect any part of body & 178 & 71.8 \\
\hline \multicolumn{3}{|l|}{ What are the symptoms of diabetes mellitus? } \\
\hline Frequent hunger & 216 & 87.1 \\
\hline Frequent thirst & 208 & 83.9 \\
\hline Frequent urination & 108 & 43.6 \\
\hline Weight loss & 50 & 20.2 \\
\hline High blood sugar & 218 & 87.9 \\
\hline Blurred vision & 176 & 70.9 \\
\hline Feeling of weakness & 221 & 89.1 \\
\hline Slow healing of cuts and wounds & 30 & 12.1 \\
\hline \multicolumn{3}{|l|}{ What are the risk factors of diabetes mellitus? } \\
\hline Age & 82 & 33.1 \\
\hline Genetic/family history of diabetes mellitus & 192 & 77.4 \\
\hline Lack of physical exercise & 230 & 92.7 \\
\hline Low nutrient intake & 99 & 39.9 \\
\hline Obesity & 111 & 44.8 \\
\hline Pregnancy & 50 & 20.2 \\
\hline Sleep duration & 33 & 13.3 \\
\hline \multicolumn{3}{|l|}{ What are the complications of diabetes mellitus? } \\
\hline Blindness & 166 & 66.9 \\
\hline Heart problem & 200 & 80.6 \\
\hline Kidney problem & 86 & 34.7 \\
\hline Brain diseases & 206 & 83.1 \\
\hline \multicolumn{3}{|l|}{ What are control and management methods of DM? } \\
\hline Insulin injection is available for control and management of DM & 248 & 100 \\
\hline Regular Exercise & 248 & 100 \\
\hline Weight reduction & 196 & 79 \\
\hline Practices healthy diet & 226 & 91.1 \\
\hline
\end{tabular}




\subsubsection{Attitude of participants towards diabetes mellitus}

Regarding the attitude of the respondents, the present study revealed that $168(67.7 \%)$ of respondents said as they do not mind if others know that they have diabetes mellitus, and 207(83.5\%) were agreed that it is important for family members should be screened for DM. Additionally, among the study respondents, 130(52.4\%) of them agreed that Diabetes mellitus seriously affect daily activities. However, around 120(48.4\%) were think as diabetes is a communicable disease and they don't know the difference between communicable and diabetes family history (Table 5).

Table 5 Frequency distribution of respondents of attitude towards diabetes mellitus among type 2 diabetic patients, Ambo university referral hospital, Oromia, Ethiopia, 2019

\begin{tabular}{|c|c|c|c|}
\hline Variables & Response & $\mathbf{N}$ & $\%$ \\
\hline \multirow[t]{3}{*}{ I don't mind if others know that I am with diabetes mellitus } & Agree & 168 & 67.7 \\
\hline & Disagree & 76 & 30.7 \\
\hline & I don't know & 4 & 1.6 \\
\hline \multirow[t]{3}{*}{ It is important that you should be examined for diabetes mellitus } & Agree & 218 & 87.9 \\
\hline & Disagree & 24 & 9.7 \\
\hline & I don't know & 6 & 2.4 \\
\hline It is important for family members should be screened for diabetes & Agree & 207 & 83.5 \\
\hline \multirow[t]{2}{*}{ Mellitus } & Disagree & 25 & 10.1 \\
\hline & I don't know & 16 & 6.5 \\
\hline \multirow[t]{3}{*}{ Family support is important in dealing with diabetes mellitus } & Agree & 248 & 100 \\
\hline & Disagree & 0 & 0.00 \\
\hline & I don't know & 0 & 0.00 \\
\hline \multirow{3}{*}{$\begin{array}{l}\text { We should follow avoiding of consumption of too much sugar for } \\
\text { controlling of DM }\end{array}$} & Agree & 218 & 87.9 \\
\hline & Disagree & 30 & 12.1 \\
\hline & I don't know & 0 & 0.00 \\
\hline \multirow[t]{3}{*}{ Diabetes mellitus seriously affect daily activities } & Agree & 130 & 52.4 \\
\hline & Disagree & 51 & 20.6 \\
\hline & I don't know & 67 & 27 \\
\hline \multirow[t]{3}{*}{ Physical activity prevent risk of DM } & Agree & 188 & 75.8 \\
\hline & Disagree & 0 & 0.00 \\
\hline & I don't know & 60 & 24.2 \\
\hline \multirow[t]{3}{*}{ Maintaining a healthy body weight is important in management of DM } & Agree & 115 & 46.4 \\
\hline & Disagree & 0 & 0.00 \\
\hline & I don't know & 133 & 53.6 \\
\hline \multirow[t]{3}{*}{ Diabetes Mellitus is a communicable disease } & Agree & 120 & 48.4 \\
\hline & Disagree & 92 & 37.1 \\
\hline & I don't know & 36 & 14.5 \\
\hline \multirow[t]{3}{*}{ Sleep duration can affect blood glucose } & Agree & 15 & 6.1 \\
\hline & Disagree & 126 & 50.8 \\
\hline & I don't know & 107 & 43.2 \\
\hline
\end{tabular}

\subsubsection{Practice towards diabetes mellitus}

The present study result shows that majority of the study participants $203(81.8 \%)$ did not have meal plans to eat ahead and also $157(63.3 \%$ ) have never visited dietitians or nutrition and other related professionals for their diet plan. From total respondents $157(63.3 \%$ ) of them reported that they frequently use fruit and vegetable while others consume fruits and vegetables less frequently. Around, 106(42.7\%) of respondents were not using fatty foods at all and the rest were using fatty foods less frequently. Again $65(26.2 \%)$ of participants were frequently practicing 30 - 60 minutes physical activity daily. Additionally $167(67.3 \%)$ of respondents frequently practice checking of blood sugar regularly (Table. 6). 
Table 6 Frequency distribution of respondents practice towards diabetes mellitus among type 2 diabetic patients, Ambo university referral hospital, Oromia, Ethiopia, 2019

\begin{tabular}{lccc}
\hline Variables & \multicolumn{3}{c}{ Response status of respondents } \\
& Not at all, & Less frequently, n & Frequently, \\
& $\mathrm{n}(\%)$ & $(\%)$ & $\mathrm{n}(\%)$ \\
\hline Do you have meal plan? & $203(81.8 \%)$ & $45(18.2 \%)$ & $0(0 \%)$ \\
Have you ever visited a dietitian/ nutritionist to consult & & & \\
about your diet plan? & $157(63.3 \%)$ & $91(36.7 \%)$ & $0(0 \%)$ \\
Do you consume fruit and vegetable? & $0(0 \%)$ & $91(36.7 \%)$ & $157(63.3 \%)$ \\
Do you consume of fatty foods? & $106(42.7 \%)$ & $107(43.2 \%)$ & $35(14.1 \%)$ \\
Did you drink alcohol & $187(75.4 \%)$ & $41(16.5 \%)$ & $20(8.1 \%)$ \\
Did you smoke tobacco & $238(95.9 \%)$ & $11(4.4 \%)$ & $0(0 \%)$ \\
Do you do 30 - 60 minutes physical activity daily? & $41(16.5 \%)$ & $142(57.3 \%)$ & $65(26.2 \%)$ \\
Have you meet sport professional for your physical activity & $217(87.5 \%)$ & $31(12.5 \%)$ & $0(0 \%)$ \\
Do you participate in maintaining your healthy weight? & $76(30.6 \%)$ & $107(43.2 \%)$ & $65(26.2 \%)$ \\
Do you check your blood sugar regularly & $0(0 \%)$ & $81(32.7 \%)$ & $167(67.3 \%)$ \\
\hline
\end{tabular}

\subsubsection{General KAP status of participants toward diabetes}

Based on the $75 \%$ of total score for knowledge (21), attitude (7.5) and practice (7.5), this study revealed that, $111(44.8 \%), 117(47.2 \%)$ and $121(48.8 \%)$ of respondents were differentiated as not knowledgeable, poor attitude level and having poor practice toward DM (figure 1).

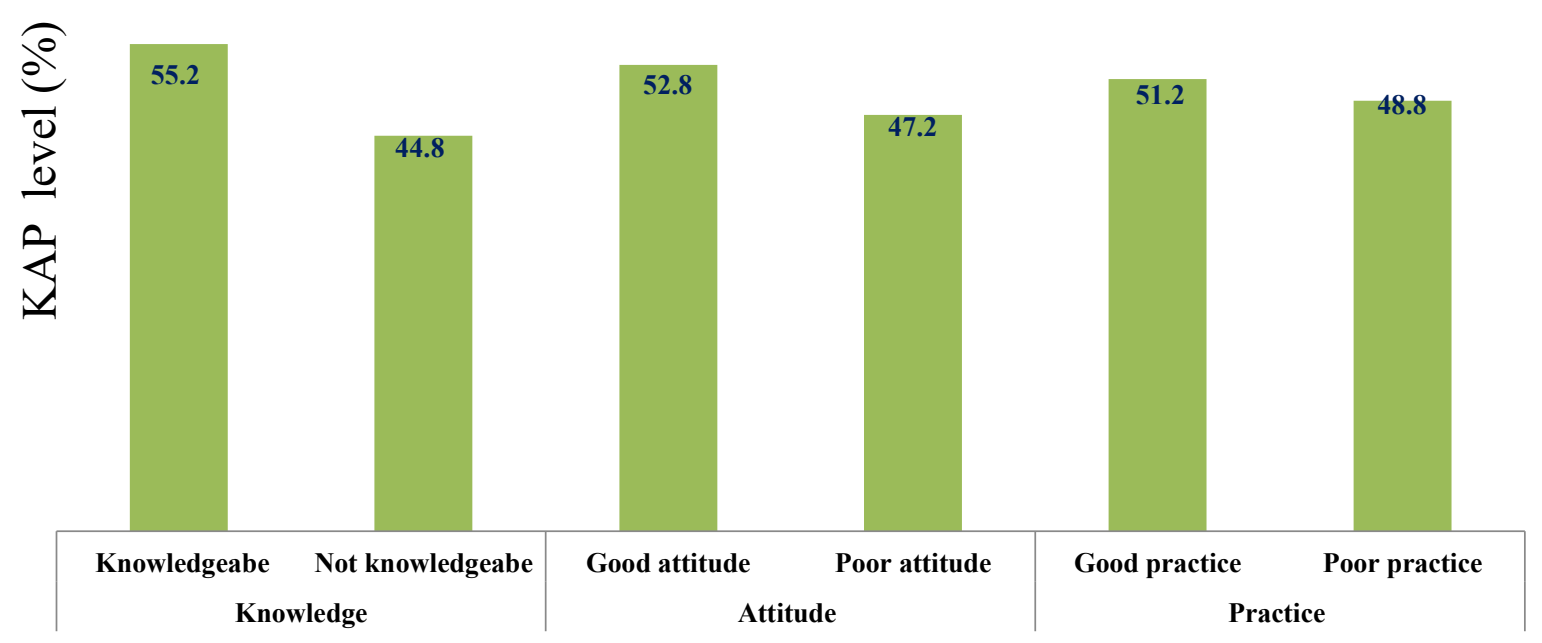

Figure 2 General status of respondents Knowledge, attitude and practice toward diabetes among type 2 diabetic patients, Ambo university referral hospital, Oromia, Ethiopia

\subsection{Factors Associated with Knowledge, Attitude and Practice}

\subsubsection{Factors associated with participant's knowledge level towards diabetes mellitus}

Table 7 below shows that age, sex, place of residence, level of education, occupational status, monthly income category, family history of diabetes and duration of living with DM showed significant associations with knowledge level of respondents. (Table 7).

\subsubsection{Factors associated with participant's attitude level towards diabetes mellitus}

Table 8 below shows that, age, place of residence, educational level, average monthly family income, family history, duration of diabetes and knowledge level showed significant associations with respondent's attitude. In the multivariable logistic regression analysis, young adults were 7.5 times $(\mathrm{AOR}=7.5, \mathrm{CI}=1.84,30.79)$ more likely to have positive attitude to DM than those from older adults. Respondents from rural areas were $80.5 \%$ (AOR= $0.195, \mathrm{CI}=0.107,0.353$ ) less likely to have positive attitude to DM than those from urban areas. Also subjects those not having formal education were $94.7 \%(\mathrm{AOR}=0.053, \mathrm{CI}=0.012,0.239)$, those with primary education level were $93.2 \%(\mathrm{AOR}=0.068, \mathrm{CI}=0.013,0.175)$ less likely to have positive attitude towards $\mathrm{DM}$ than those with higher education level. In the case income level, individuals earning average monthly family income of $\leq 500$ birr were $74.6 \%(\mathrm{AOR}=.254, \mathrm{CI}=0.18,0.69), 501-1000$ birr were $72.5 \%(\mathrm{AOR}=0.275, \mathrm{CI}=0.13,0.55)$ less likely to have positive attitude towards DM compared to those earned $>2000$ birr. Additionally, participants with no diabetes history were $67.6 \%(\mathrm{AOR}=0.324, \mathrm{CI}=0.15,0.70)$ less likely to have positive attitude compared to those 
having family diabetes history. In the same way participants those were diabetic for 1-6 months were $93 \%$ $(\mathrm{AOR}=0.07, \mathrm{CI}=0.01,0.28)$ less likely to have positive attitude towards DM compared to those were diabetic for 13 and above months. Furthermore being not knowledgeable about diabetes had $77.3 \%$ decreases in their positive attitude level about diabetes as compared to those who were knowledgeable (Table 8).

Table 7 Bi-variable and multivariable logistic regression predicting diabetes mellitus related knowledge level among study participants, Ambo university referral hospital, Oromia, Ethiopia, 2019

\begin{tabular}{|c|c|c|c|c|c|c|c|}
\hline \multirow[t]{2}{*}{ Variable } & \multirow[t]{2}{*}{ Level } & \multirow{2}{*}{$\begin{array}{l}\begin{array}{l}\text { Not } \\
\text { knowledgeable }\end{array} \\
\text { n (\%) }\end{array}$} & \multirow{2}{*}{$\begin{array}{r}\text { Knowledgeable } \\
\text { n (\%) }\end{array}$} & \multicolumn{4}{|c|}{ Indicators of relationship } \\
\hline & & & & p-value & COR $(95 \% \mathrm{CI})$ & p-value & AOR (95\%CI) \\
\hline \multirow[t]{3}{*}{ Age } & young adults & $6(21.4)$ & $22(78.6)$ & $.002 * *$ & $6.87(1.97-23.88)$ & $.043^{*}$ & $6.4(1.06-38.61)$ \\
\hline & middle-aged adults & $90(45.7)$ & $107(54.3)$ & .082 & $2.22(0.90-5.49)$ & .099 & $2.70(.83-8.81)$ \\
\hline & older adults & $15(65.2)$ & $8(34.8)$ & 1 & & & \\
\hline \multirow[t]{2}{*}{ Sex } & Male & $63(38.9)$ & $99(61.1)$ & $.011 *$ & $1.98(1.16-3.37)$ & $.001^{* *}$ & $8.46(2.5-28.63)$ \\
\hline & Female & $48(55.8)$ & $38(44.2)$ & 1 & & & \\
\hline \multirow[t]{2}{*}{ Residence } & Rural & $81(77.1)$ & $24(22.9)$ & $.000 * * *$ & $.07(.04-.14)$ & $.000 * * *$ & $.12(.04-.39)$ \\
\hline & Urban & $30(21.0)$ & $113(79.0)$ & 1 & & & \\
\hline \multirow[t]{2}{*}{ Family size } & $\leq 5$ & $40(46.0)$ & $47(54.0)$ & .777 & $.92(.54-1.56)$ & - & - \\
\hline & $>5$ & $71(44.1)$ & $90(55.9)$ & 1 & & & \\
\hline \multirow[t]{4}{*}{ Living status } & Living alone & $2(15.4)$ & $11(84.6)$ & $.004 * *$ & $18.33(2.52-133.26)$ & .239 & $.19(.01-2.96)$ \\
\hline & $\begin{array}{l}\text { Living } \\
\text { wife/husband }\end{array}$ & $99(44.6)$ & $123(55.4)$ & $.034 *$ & $4.14(1.11-15.45)$ & .825 & $1.2(.23-6.16)$ \\
\hline & Living with others & $10(76.9)$ & $3(23.1)$ & 1 & & & \\
\hline & Married & $98(44.5)$ & $122(55.5)$ & .082 & $3.32(.85-12.84)$ & - & - \\
\hline \multirow[t]{3}{*}{ Marital status } & Divorced & $3(75.0)$ & $1(25.0)$ & .930 & $.88(.06-12.25)$ & - & - \\
\hline & Single & $2(15.4)$ & $11(84.6)$ & .109 & $14.66(1.97-109.20)$ & - & - \\
\hline & Widowed & $8(72.7)$ & $3(27.3)$ & 1 & & & \\
\hline \multirow{4}{*}{$\begin{array}{l}\text { Education } \\
\text { level }\end{array}$} & No formal education & $48(84.2)$ & $9(15.8)$ & $.000 * * *$ & $.009(.002-.034)$ & $.000^{* *}$ & $.017(.001, .06)$ \\
\hline & Primary education & $46(52.9)$ & $41(47.1)$ & $.000 * * *$ & $.041(.012-.141)$ & $.001 * *$ & $.043(.004, .26)$ \\
\hline & Secondary education & $14(38.9)$ & $22(61.1)$ & $.000 * * *$ & $.073(.019-.276)$ & .155 & $.177(.017,1.88)$ \\
\hline & Higher education & $3(4.4)$ & $65(95.6)$ & 1 & & & \\
\hline \multirow{4}{*}{$\begin{array}{l}\text { Occupational } \\
\text { status }\end{array}$} & Housewife only & $22(68.8)$ & $10(31.3)$ & $.000 * * *$ & $.091(.035-.233)$ & $.004 * *$ & $.046(.004, .320)$ \\
\hline & Farmer & $65(70.7)$ & $27(29.3)$ & $.000 * * *$ & $.083(.040-.172)$ & .902 & $3.2(.064,22.65)$ \\
\hline & Merchants & $10(25.0)$ & $30(75.0)$ & .275 & $.60(.240-1.50)$ & $.001 * *$ & $.061(.004, .26)$ \\
\hline & Employed & $14(16.7)$ & $70(83.3)$ & 1 & & & \\
\hline \multirow[t]{4}{*}{ Family income } & $\leq 500 \mathrm{ETB}$ & $46(63.0)$ & $27(37.0)$ & $.000 * * *$ & $.18(.09-.36)$ & $.000 * * *$ & $.198(.08-.45)$ \\
\hline & $501-1000$ ЕТВ & $37(60.7)$ & $24(39.3)$ & $.000 * * *$ & $.20(.10-.41)$ & $.015^{*}$ & $.320(.12-.80)$ \\
\hline & $1001-2000$ ЕТВ & $5(27.8)$ & $13(72.2)$ & .730 & $.81(.26-2.54)$ & .827 & $1.15(.30-4.33)$ \\
\hline & $>2000 \mathrm{ETB}$ & $23(24.0)$ & $73(76.0)$ & 1 & & & \\
\hline \multirow{2}{*}{$\begin{array}{l}\text { DM history of } \\
\text { the family }\end{array}$} & No diabetic history & $47(61.0)$ & $30(39.0)$ & $.001 * *$ & $.38(.22-.66)$ & $.005^{* *}$ & $.29(.12-.68)$ \\
\hline & $\begin{array}{l}\text { Having diabetic } \\
\text { history }\end{array}$ & $64(37.4)$ & $107(62.6)$ & 1 & & & \\
\hline Duration & $1-6$ months & $28(77.8)$ & $8(22.2)$ & $.000 * * *$ & $.127(.05-.29)$ & $.000 * * *$ & $.149(.05-.40)$ \\
\hline \multirow[t]{2}{*}{ DM } & $7-12$ months & $30(75.0)$ & $10(25.0)$ & $.000^{* * *}$ & $.148(.06-.32)$ & $.000^{* * *}$ & $.097(.04 .23)$ \\
\hline & 13 months \& above & $53(30.8)$ & $119(69.2)$ & 1 & & & \\
\hline
\end{tabular}


Table 8 Bi-variable and multivariable logistic regression predicting diabetes mellitus related attitude level among study participants, Ambo university referral hospital, Oromia, Ethiopia, 2019

\begin{tabular}{|c|c|c|c|c|c|c|c|}
\hline \multirow[t]{2}{*}{ Variable } & \multirow[t]{2}{*}{ Level } & \multirow{2}{*}{$\begin{array}{c}\text { Poor attitude } \\
\mathbf{n}(\%)\end{array}$} & \multirow{2}{*}{$\begin{array}{r}\begin{array}{l}\text { Good } \\
\text { attitude }\end{array} \\
n(\%)\end{array}$} & \multicolumn{4}{|c|}{ Indicators of relationship } \\
\hline & & & & p-value & COR (95\% CI) & p-value & $\operatorname{AOR}(95 \% \mathrm{CI})$ \\
\hline \multirow[t]{3}{*}{ Age } & young adults & $9(32.1)$ & $19(67.9)$ & $.010^{*}$ & $\begin{array}{r}4.82(1.46- \\
15.87)\end{array}$ & $.005 * *$ & $7.5(1.84-30.79)$ \\
\hline & middle-aged adults & $92(46.7)$ & $105(53.3)$ & $.044^{*}$ & $2.60(1.02-6.62)$ & .193 & $2.06(.69-6.13)$ \\
\hline & older adults & $16(69.6)$ & $7(30.4)$ & 1 & & & \\
\hline \multirow[t]{2}{*}{ Sex } & Male & $75(46.3)$ & $87(53.7)$ & .703 & $1.10(.65-1.86)$ & & - \\
\hline & Female & $42(48.8)$ & $44(51.2)$ & 1 & & & \\
\hline \multirow[t]{2}{*}{ Residence } & Rural & $75(46.3)$ & $87(53.7)$ & $.000 * * *$ & $.195(.113-.337)$ & $.000 * * *$ & $.195(.107-.353)$ \\
\hline & Urban & $42(48.8)$ & $44(51.2)$ & 1 & & & \\
\hline \multirow[t]{4}{*}{ Education level } & No formal education & $40(70.2)$ & $17(29.8)$ & $.000 * * *$ & $.073(.030-.176)$ & $.000 * * *$ & $.053(.012-.239)$ \\
\hline & Primary education & $60(69.0)$ & $27(31.0)$ & $.000 * * *$ & $.078(.035-.174)$ & $.000^{* * * *}$ & $.068(.013-.175)$ \\
\hline & Secondary education & & & .535 & $.714(.247-$ & .496 & $.626(.162-$ \\
\hline & Higher education & $\begin{array}{r}7(19.4) \\
10(14.7)\end{array}$ & $\begin{array}{l}29(80.6) \\
58(85.3)\end{array}$ & (.0נ & 2.069) & & 2.414) \\
\hline \multirow[t]{4}{*}{ Occupational status } & Housewife only & $30(93.8)$ & $2(6.3)$ & $.000 * * *$ & $.013(.003-.062)$ & .429 & $.35(.02-4.65)$ \\
\hline & Farmer & $59(64.1)$ & $33(35.9)$ & $.000 * * *$ & $.112(.055-.229)$ & .174 & $6.16(.44-85.08)$ \\
\hline & Merchants & $14(35.0)$ & $26(65.0)$ & $.025^{*}$ & $.371(.156-.884)$ & .072 & $6.92(.84-56.95)$ \\
\hline & Employed & $14(16.7)$ & $70(83.3)$ & 1 & & & \\
\hline \multirow[t]{4}{*}{ Marital status } & Married & $101(46.1)$ & $118(53.9)$ & $.036^{*}$ & $\begin{array}{r}5.25(1.11- \\
24.89)\end{array}$ & .212 & $2.86(.55-14.88)$ \\
\hline & Divorced & $4(80)$ & $1(20.0)$ & .931 & $1.12(.07-16.30)$ & .696 & $.56(.03-9.83)$ \\
\hline & Single & $3(23.1)$ & $10(76.9)$ & & $\begin{array}{r}15(2.02- \\
111.17)\end{array}$ & .133 & $5.14(.61-43.71)$ \\
\hline & Widowed & $9(81.8)$ & $2(18.2)$ & 1 & & & \\
\hline \multirow[t]{3}{*}{ Living status } & Living alone & $3(23.1)$ & $10(76.9)$ & $.004 * *$ & $\begin{array}{r}18.33(2.52- \\
133.26)\end{array}$ & .096 & $6.04(.72-50.23)$ \\
\hline & $\begin{array}{l}\text { Living with wife or } \\
\text { husband }\end{array}$ & $103(464)$ & $119(536)$ & $.018^{*}$ & $\begin{array}{r}6.35(1.37- \\
29.33)\end{array}$ & .153 & $3.26(.65-16.56)$ \\
\hline & Living with others & $11(84.6)$ & $2(15.4)$ & 1 & & & \\
\hline \multirow[t]{4}{*}{ Religion } & Orthodox & $95(58.6)$ & $67(41.4)$ & .124 & $.050(.006-.39)$ & & - \\
\hline & Protestant & $18(32.1)$ & $38(67.9)$ & .078 & $.151(.018-1.23)$ & & - \\
\hline & Waaqeffataa & $3(20)$ & $12(80)$ & .304 & $.286(.026-3.12)$ & & - \\
\hline & Muslim & $1(6.7)$ & $14(93.3)$ & 1 & & & \\
\hline \multirow[t]{4}{*}{ Family income } & $\leq 500$ ETB & $42(57.5)$ & $31(42.5)$ & $.000 * * *$ & $.31(.16-.60)$ & $.002 * *$ & $.254(.18-.69)$ \\
\hline & $501-1000$ ЕТВ & $38(62.3)$ & $23(37.7)$ & $.000 * * *$ & $.26(.13-.51)$ & $.000 * * *$ & $.275(.13-.55)$ \\
\hline & $1001-2000$ ЕTB & $8(44.4)$ & $10(55.6)$ & .241 & $.54(.19-1.51)$ & .274 & $.55(.19-1.59)$ \\
\hline & $>2000 \mathrm{ETB}$ & $29(30.2)$ & $67(69.8)$ & 1 & & & \\
\hline \multirow{2}{*}{$\begin{array}{l}\text { Family diabetic } \\
\text { history }\end{array}$} & No diabetic history & $49(63.6)$ & $28(36.4)$ & $.001 * *$ & $.37(.21-.65)$ & $.004 * *$ & $.324(.15-.70)$ \\
\hline & Having diabetic history & $68(39.8)$ & $103(60.2)$ & 1 & & & \\
\hline \multirow[t]{3}{*}{ Duration of DM } & $1-6$ months & $33(91.7)$ & $3(8.3)$ & $.000 * * *$ & $.065(.019-.222)$ & $.000^{* * *} *$ & $.07(.01-.28)$ \\
\hline & $7-12$ months & $12(30)$ & $28(70)$ & .170 & $1.68(.80-3.52)$ & .438 & $1.50(.53-4.23)$ \\
\hline & 13 months \& above & $72(41.9)$ & $100(58.1)$ & 1 & & & \\
\hline \multirow[t]{2}{*}{ Knowledge } & Not Knowledgeable & $77(69.4)$ & $34(30.6)$ & $.000 * * *$ & $.182(.105-.314)$ & $.000^{* * *}$ & $.227(.127-406)$ \\
\hline & Knowledgeable & $40(29.2)$ & $97(70.8)$ & 1 & & & \\
\hline
\end{tabular}

$* \mathrm{p}<0.05 ; * * \mathrm{p}<0.01 ; * * * \mathrm{p}<0.001$

\subsubsection{Factors associated with participants practice level towards diabetes mellitus}

Table below shows that as age, place of residence, level of education, average monthly family income, diabetic history of family, duration of DM, diabetes knowledge level, and diabetes attitude levels showed significant associations with practice towards DM. In the multivariable analysis, individuals who were young adults were 9.34 times $(\mathrm{AOR}=9.34, \mathrm{CI}=1.53,56.68)$ more likely to practice compared to those older adults. The other was respondents from rural areas were $84.4 \%(\mathrm{AOR}=0.156, \mathrm{CI}=0.030,0.815)$ less likely to practice than those from urban areas. Individuals those have no formal education were $81.8 \%(\mathrm{AOR}=0.182, \mathrm{CI}=0.056,0.589)$ less likely to practice than those with higher educational level. Also this study revealed that subjects those having $\leq 500$ Ethiopian birr average monthly family income were $67.9 \%(\mathrm{AOR}=0.321, \mathrm{CI}=0.12,0.81)$ less likely to practice compared to those having $>2000$ Ethiopian birr average monthly family income. Individuals with no diabetic history were $61.9 \%(\mathrm{AOR}=0.381, \mathrm{CI}=0.218,0.673)$ less likely to practice than those of having family diabetes history. In the case of DM duration participants those were diabetic for 1-6 months were 58.6\% times $(\mathrm{AOR}=$ $0.414, \mathrm{CI}=0.191,0.897)$ less likely to practice than those were diabetic for 13 and above months. In terms diabetes knowledge level, having poor diabetes knowledge had $72.6 \%(\mathrm{AOR}=0.274, \mathrm{CI}=0.134,0.560)$ less likely to practice than those having good diabetes knowledge. Lastly, having poor attitude had $60.7 \%(\mathrm{AOR}=0.393, \mathrm{CI}=$ $0.206,0.753$ ) less likely to practice than those having good attitudes towards diabetes (Table 9). 
Table 9 Bi- variable and multivariable logistic regression predicting diabetes mellitus related practice level among study participants, Ambo University referral hospital, Oromia, Ethiopia, 2019

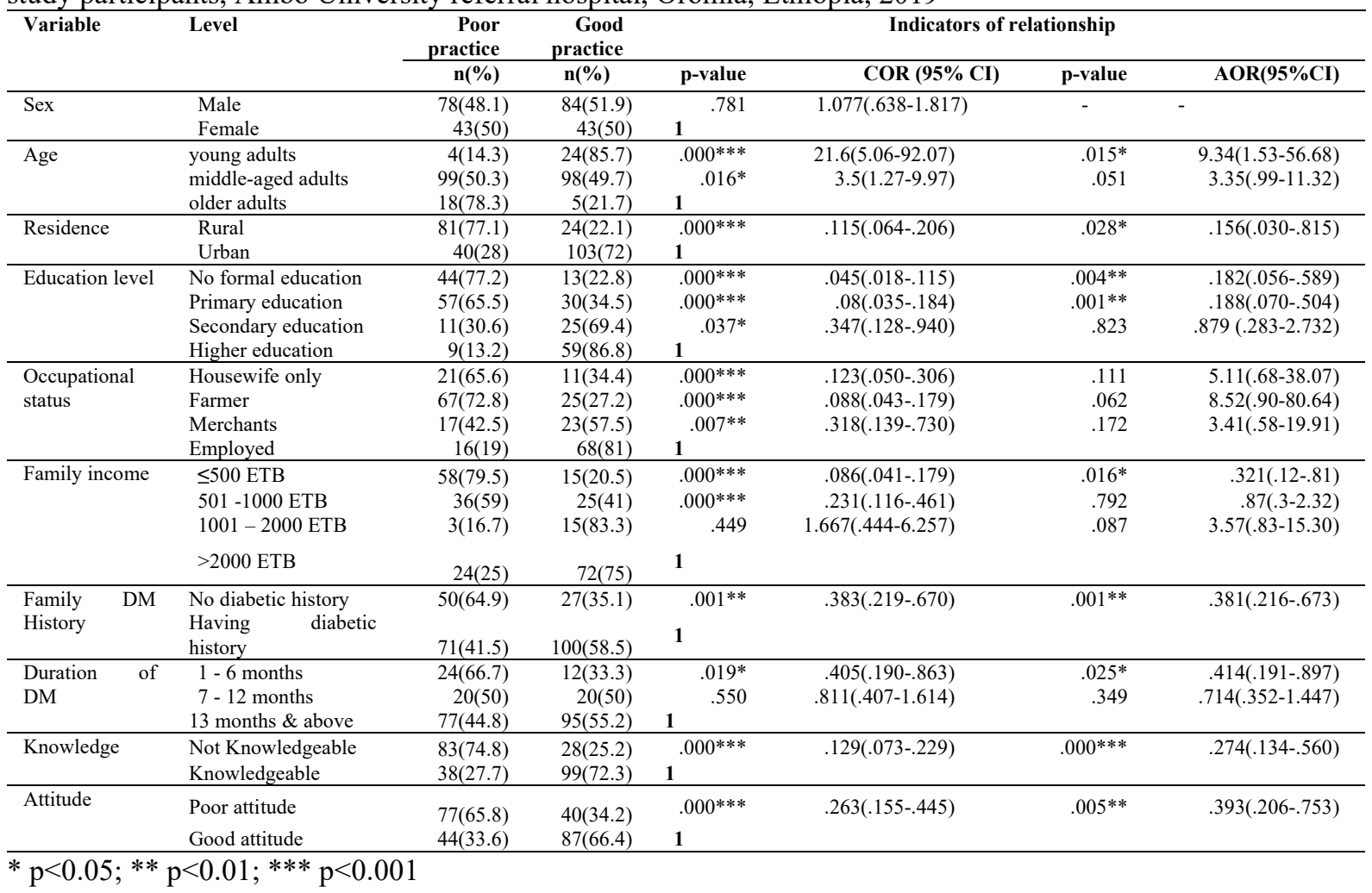

\subsection{Discussion}

Diabetes is a chronic illness and long-term metabolic disorder that drastically affecting health of the population throughout the world. T2DM is the most common form of diabetes mellitus that affecting adults. This study revealed that $(55.2 \%)$ of respondents had a good knowledge. This finding was higher than the study done in Malaysia (41.9\%) (Minhat et al., 2014) and inBangladesh (17\%) (Fatema et al., 2017). This may be due to the study was hospital-based, where the information was accessible.

According to the findings of this study, young adults and middle aged adults were 6.4 times more likely to have knowledge than that of older adults. This finding is consistent with the results reported by Shrestha et al., (2015) in Central Nepal patients, Jasper et al., (2014) in Nigerian and Gillani et al., (2018) in Pakistan, that younger age had significantly higher diabetes knowledge score than older participants. This is may be old age, with deteriorating cognitive function, is considered as a barrier to diabetes education whereas younger patients might have higher motivation and adaptability towards their disease. Moreover, in this study, males were 8.46 times more likely to have knowledge than females. This finding is similar to a study conducted by Saleh et al.,(2016)in Bangladish but contrast with a study done by Gillani et al., (2018)in Pakistan where women were significantly more aware of DM than males.

Regarding the residence, respondents who living in rural area were $88 \%$ less knowledgeable than those from urban and this result is in line with studies done by El-Khawaga et al., (2015) in Egypt and Gillani et al.,(2018) in Pakistan, in which urban residents were more knowledgeable. This may be described by the high availability and accessibility of different health based information in urban compared to rural or gap of information is available in rural compared to urban.

The present study showed significant association between levels of education and level of knowledge among the respondents. Participants who had no formal educations were $98.3 \%$ and those with primary education level were $95.7 \%$ less knowledgeable than those with college and above education level. This result is similar to other studies done by Deepa et al., (2017) in India and Feleke et al., (2013) in Ethiopia. Almost certainly, it is because of respondents who had higher education would have the chance to get information which make them more aware about diabetes. Concerning occupational status, individuals who had housewife only were 95.4\% less likely knowledgeable than those who were government and private employed. This may be due to a gap in exposure to information compared to those who had other works.

This study revealed that participants earning average monthly income of $\leq 500$ Ethiopian birr were $80.2 \%$ less likely to have knowledge compared to those earning $>2000$ Ethiopian birr. This findings were supported by studies 
done in Ethiopia by Kassahun et al., 2017, Pakistan (Gillani et al., 2018) and Malaysia (Minhat et al., 2014) This might be due to having higher income level will help to access and afford energy density food which might cause diabetes problem among those people.

According to the findings of this study, participants with no diabetic history were $71 \%$ less likely to have knowledge than those having diabetic history. Besides, respondents those with $\leq 6$ months disease duration were $85.1 \%$ less likely to have diabetes knowledge than those with 13 months and above durations of diseases. This finding is supported by studies conducted by Rahaman et al., (2017) in Dhaka and Feleke et al., (2013) in Ethiopia. This may be due to having diabetes history and living long time with the disease provide more exposure to the information regarding the disease and there will be the chance to communicate with health professionals during their health follow up.

This this study also found that participants who were young and middle adults had better attitude score than elder adults. This result is supported by studies done by Rahaman et al., (2017) in Dhaka, Gautam et al., (2015) in Nepal, Maretha et al., (2018) in South Africa. This may be due to higher cognitive performance among younger adult than older adults.

Regarding place of residences, respondents who lived in rural were $80.5 \%$ less likely to have positive attitude towards DM than those from urban. This result is in line with Fatema et al., 2017 in Bangladesh.This may be urban residents may got chance to gate more nutrition and health information from mass media compared those who lived in rural.

Moreover, respondents who had no formal education were $94.7 \%$ less likely in attitude level as compared to those who had higher educational levels. Besides, this finding is in line withthe study done in Ethiopia by Kassahun et al., (2017). Probably, it is because of respondents who had higher education would have the chance to get adequate information about diabetes compared those who had no formal education.

Furthermore, this study showed that both diabetes history and duration of the disease were significantly associated with attitude level. Participants who had a 1-6 months DM duration were 93\% fold decreased in attitude level. This result is consistent with the study in Dhaka (Rahaman et al., 2017) in which Patients with long duration of diabetes had better attitude score than those who had short duration of diabetes. This is might be due to getting and more understanding of informationthrough experience.

Additionally, being not knowledgeable about diabetes had $77.3 \%$ decreases in their positive attitude level compared to those who were knowledgeable. This means that "the higher their knowledge, the better their attitude. These finding is supported by the ideas about positive correlation between knowledge and good attitude observed among participants in studies done in Riyadh, Saudi Arabia (Salem et al., 2018) and in Ethiopia (Kashahun et al., 2017).

In the sense of practice towards DM this study result shows that $48.2 \%$ of participants were having poor practice towards diabetes mellitus. different socio demographic factors including age, place of residence, education level, income level, family history of the disease, duration of the disease, knowledge and attitude level were identified as determining factors for participants attitude level towards diabetes. In addition, this study, younger participants had better practice score than their elders. The finding is supported by studies done in by Felekeet al., (2013) and in Daka by Rahaman et al., (2017).

Moreover, regarding residences, respondents who lived in rural were $84.4 \%$ less likely to have practice towards DM than those from urban. This result is in touch with the study conducted by Niguse et al., (2019) in Ethiopia in which participants from urban were better practicing compared to those from rural.

Concerning, education level, respondents who had no formal education were $81.8 \%$ less practiced compared to those who had higher educational levels. This finding is consistent with the studies conducted inSaudi Arabia by Salem et al.,(2018) andMohammadi et al., (2015). This might be literacy may be the most important in diabetes management.

Furthermore, this study showed that participants with no diabetic history were $61.9 \%$ less likely to have better practice than those having diabetic history. In addition, respondents who were diabetic for 1-6 months were 58.6\% decreased in practice level. This result is in line with studies conducted by Salem, et al., (2018); Niroomand et al., (2015) and (Rahaman et al., 2017) in which found that diabetic history was associated with practice level towards diabetes mellitus.

Finally, this study revealed that both knowledge level and attitude level were among the factors affecting of practicing level. According to this study, participants who had poor knowledge and poor attitude level were less likely to practice compared to their counterparts. This result is consistent with the study conducted in Ethiopia (Kassahun et al., 2017). This indicates that having good KAP towards diabetes mellitus can improve practice level.

\section{Conclusion}

This study concluded that about $44.8 \%, 47.2 \%$ and $48.8 \%$ of respondents were differentiated as not knowledgeable, having poor attitude and poor practice towards diabetes mellitus, respectively. Additionally the current study revealed that age, place of residence, level of education, average monthly income, family history of diabetes and 
duration of living with DM were factors affecting KAP towards diabetes. Both sex and occupational status were affecting the knowledge level, but not attitude and practice. Furthermore, knowledge level was affecting booth attitude and practice level of participants in the study area at the significance level of $(p<0.05)$. Generally, lack of knowledge, poor attitude, and poor practice level were found in this cross sectional study. Therefore, a structured nutrition and health education should be given for T2DM patients through community based behavioral change to assist and improve level of knowledge of DM patients, since knowledge is an important factor to influence someone's attitude and practice. Nutritionists/health extension workers should also give nutrition education through mass media like TV, radio to overcome the problems of KAP regarding DM.

\section{References}

Abdulkadir M, Adem H. 2014. Assessment of Knowledge, Attitude and Practices Regarding Life Style Modification among Type 2diabetic Mellitus Patients Attending Adama Hospital Medical College, Oromia Region, Ethiopia. Global Journal of Medical Research: B Pharma, Drug Discovery, Toxicology and Medicine.

Achenef A et al. 2015. Knowledge and Attitude About Diabetes Mellitus and Its Associated Factors Among People in DebreTabor Town,Northwest Ethiopia: Cross Sectional Study. Science Journal of Public Health, pp. 199-209.

ADA. 2010. American Diabetes Association. Diagnosis and classification of diabetes mellitus. Diabetes Care; 33:S62-S69.

Beke et al. 2013. A cross-sectional survey on the lifestyle and health seeking of Basotho patients with diabetes. South African Family Practice, 55(2), 190-195.

Belayneh A, Teklie H, Tadesse M, Defar A, Getachew T, Amenu K, et al. 2015. Pattern and Trend of Medical Admissions of Patients of Chronic NonCommunicable Diseases in Selected Hospitals in Addis Ababa, Ethiopia. American Scientific Research Journal for Engineering, Technology, and Sciences: pp. 33-48.

CDC.2012. National diabetes fact sheet: National estimates and general information on diabetes and pre-diabetes in the United States.

Cho N, W. D. 2013. International diabetes federation atlas report: Global burden of diabetes Belgium, Brussels community in northern Nigeria. Nigerian Journal of Medicine, pp. 414-416.

Deepa L, Pallavi M, Madhavi R, Muninarayan C., et al.2017. Assessment of awareness and practice of foot care and physical activity among people with type 2 diabetes attending a tertiary care teaching hospital. International Journal of Community Medicine and Public Health, 4(9):3218-3223.

El-Khawaga G, Abdel-Wahab F. 2015. Knowledge, Attitudes, Practice And Compliance Of Diabetic Patients In Dakahlia, Egypt. European Journal of Research in Medical Sciences. 2056-600X

Fatema K,Hossain S, Natasha K et al. 2017. Knowledge attitude and practice regarding diabetes mellitus among Nondiabetic and diabetic study participants in Bangladesh. BMC Public Health; 17: 364

Feleke SA, Misganaw CA, Adane HT.2013. Assessment of the level and associated factors with knowledge and practice of Diabetes Mellitus among Diabetic Patients attending at FelegeHiwot Hospital, Northwest Ethiopia: Clinical Medicine Research: pp. 110-120

Gauntam B. Rathod1, Sangita Rathod, et al. 2015.Study of Knowledge, Attitude and Practice of General Population of Waghodia towards Diabetes Mellitus International Journal of Current Research and Review. pp: 63-68

Gillani et al. 2018. Knowledge, Attitudes and Practices Regarding Diabetes in the General Population: A Cross-Sectional Study from Pakistan. International Journal of Environmental Research and Public Health, 15, 1906

Guariguata L, Whiting DR, Hambleton I, Beagley J et al. 2013. Global estimates of diabetes prevalence for 2013 and projections for 2035: IDF Diabetes Atlas: Diabetes Research and Clinical Practice; pp. 137-149

Kassahun CW, Mekonen AG. 2017.Knowledge, attitude, practices and their associated factors towards diabetes mellitus among non diabetes community members of Bale Zone administrative towns, South East Ethiopia. A cross-sectional study. Peer-reviewed open access scientific journal published by the Public Library of Science. PP 1-18

Mabaso RG, O. O. 2014. Risk factors for visual impairment and blindness amongst black adult diabetics receiving treatment at Government healthcare facilities in Mopani District, Limpopo province, South Africa. African Journal of Primary Health \& Family Medicine; 6 (1), Art. \#623, 8 pages.

Maretha le Roux, Corinna W, Marianne R, Jacques R.2018.Diabetes-related knowledge, attitude and practices (KAP) of adult patients with type 2 diabetes mellitus in the Free State province, South Africa. South African Journal of Clinical Nutrition. PP:83-90

Megersa Y,et al. 2013. Prevalence of undiagnosed DM and its risk factors in selected institutions of at Bishoftu town. journal of diabetes, s12:068 
Minhat HS, HamedonTR. 2014. Understanding towards Diabetes Mellitus among Rural Adult Community in Malaysia.World Journal of Medical Sciences 11 (2): 217-221

Mohammadi S, Norimah A., Ruzita AT et al. 2015. Knowledge, Attitude and Practices on Diabetes Among Type 2 Diabetic Patients in Iran: A Cross-Sectional Study. Science Journal of Public Health. pp.520-524.

Niguse H, Belay G, Fisseha G, Desale T, Gebremedhn G. 2019.Self-care related knowledge, attitude, practice and associated factors among patients with diabetes in Ayder Comprehensive Specialized Hospital, North Ethiopia.BMC Research Notes. 2019 Jan 18;12(1):34.

Niroomand M, S. N. 2015. Diabetes knowledge, attitude and practice (KAP) study among Iranian in-patients with type-2 diabetes: A cross-sectional study. Diabetes \& Metabolic Syndrome: Clinical Research

Primanda Y, Kritpracha C, et al. 2011. Dietary Behaviors among Patients with Type 2 Diabetes Mellitus in Yogyakarta, Indonesia. Nurse Media Journal of Nursing. pp. 211-23.

Rahaman KH, Majdzadeh R, Naieni KH and Raza O. 2017. Knowledge, Attitude and Practices (KAP) Regarding Chronic Complications of Diabetes among Patients with Type 2 Diabetes in Dhaka. International Journal of Endocrinology and Metabolism.

Raymond G. Mabaso, O. A. 2016. Knowledge and practices related to diabetes mellitus among adults with diabetes in the Mopani District, Limpopo Province, South Africa. Original Research AOSIS

Saleh F, Mumu SJ , Ara F, Begum HA, Ali L .2012. Knowledge and self-care practices regarding diabetes among newly diagnosed type 2 diabetics in Bangladesh: a cross-sectional study. BioMed Central.

Salem A, Alshammari M, et al. 2018. Knowledge, Attitude, and Practice Regarding Diabetes Mellitus among General Public and Diabetic Patients in Riyadh, Saudi Arabia. Asian Journal of Pharmaceutics. Jan-Mar 2018 (Suppl). $12(1) \mid \mathrm{S} 1$

Shrestha N, Yadav SB, Joshi AM, Patel BDP, Shrestha J, Bharkher DL. 2015. Diabetes Knowledge and Associated Factors among Diabetes Patients in Central Nepal. International Journal of Collaborative Research on Internal Medicine \& Public Health.

Tadele Amente, T. B. 2014. Self Care Practice and its Predictors Among Adults with Diabetes Mellitus on Follow Up At Nekemte Hospital Diabetic Clinic, West Ethiopia. World Journal of Medicine and Medical Science, PP: 1 - 16.

WHO. 2008. Expert Consultation Geneva, December 2008. 8-11.

WHO. 2016. Global Report on Diabetes

Worku D.2010. Patterns of Diabetic Complications at Jimma University Specialized Hospital, Southwest Ethiopia. Ethiopian Journal of Health Sciences. March 2010, pp. 33-40 